\title{
Bayer Immuno-1 PSA Test
}

National Cancer Institute

\section{Source}

National Cancer Institute. Bayer Immuno-1 PSA Test. NCI Thesaurus. Code C113701.

A proprietary system designed to measure protein-complexed prostate-specific antigen

(CPSA) in the blood. 